Gesnerus 55 (1998) 205-220

\title{
Hexensalben und Liebestränke
}

Ein Beitrag zur Kulturgeschichte der Nachtschattengewächse

Jürgen Müller

\section{Summary}

The nightshades (solanaceae) were used as intoxicants since the ancient civilisations and are still in use today. Their alkaloids, atropine and scopolamine, were the major active substances of the ointments of witches, of medieval "anaesthetics", and of modern poisons for murder. In a medium dose-range the predominant symptoms are hallucinations and illusions. This explains the use of nightshades in fortune-telling and religious rituals. In higher doses the alkaloids produce coma and apnea. Scopolamine enjoyed a particular popularity as a poison for murder. In the $19^{\text {th }}$ century the nightshade alkaloids were also in clinical use.

This article focusses on the medical history of the psychosis due to intoxication with solanaceae.

\section{Zusammenfassung}

Die Nachtschattengewächse (Solanazeen) werden seit der Antike in nahezu jedem Kulturkreis als Rauschdrogen verwendet. Sie werden auch gegenwärtig noch als Rauschdrogen benutzt. Die Solanazeenalkaloide Atropin und Skopolamin waren die Hauptwirksubstanzen der Hexensalben, mittelalterlicher Narkotika und neuzeitlicher Mordgifte.

Bei einer Vergiftung tritt ein dosis- und substanzabhängiges Krankheitsbild mit zentralen und peripheren Symptomen auf. Im mittleren Dosisbereich stehen psychopathologische Halluzinationen im Vordergrund, die den früheren Einsatz zu religiösen und wahrsagerischen Zwecken erklären. Der bei hohen Dosen auftretende Atemstillstand führte dazu, dass insbe-

Dr. Jürgen Müller, IFPD, Universität Bern, Falkenplatz 3, CH-3012 Bern 
sondere Skopolamin als «Mordgift» eingesetzt wurde. Im 19. Jahrhundert wurden die Nachtschattenalkaloide auch klinisch verwendet.

Im folgenden wird eine Übersicht über die Kulturgeschichte der durch die Solanazeen hervorgerufenen Intoxikationspsychosen gegeben.

\section{Einleitung}

Angewendet in Form von Analgetika und Hypnotika, Rausch- und Wahrheitstränken, Hexensalben und Aphrodisiaka sind die Nachtschattengewächse von herausragender medizin- und kulturgeschichtlicher Bedeutung ${ }^{1}$. Noch immer werden die Solanazeen weltweit als Rauschdrogen benutzt ${ }^{2}$. Als «zentrales anticholinerges Syndrom» wird dieses jahrhundertealte, charakteristische Krankheitsbild heute mit Syndromen anderer Ätiologie vermengt ${ }^{3}$. Häufig wird die Diagnose erst toxikologisch nach dem Abklingen des akuten Stadiums gestellt. Ich nehme einen aktuellen Fall zum Anlass, die medizin- und kulturgeschichtliche Dimension der Solanazeenintoxikationen zu umreissen und eine Brücke zwischen Vergangenheit und Gegenwart zu schlagen:

Ein 30jähriger Patient trank auf einer Party von einem selbstgefertigten, ihm unbekannten Getränk, das er wegen seiner «tollen» Wirkung probieren sollte. Wenig später wurde ihm übel. Sein Gang wurde unsicher, und er sah verschwommen. Er stieg ins Auto, um nach Hause zu fahren. Von da ab konnte er sich bis zum folgenden Tag an nichts mehr erinnern. Von einem

1 Eine schöne Darstellung eines «Hexensabbats», «La danse du Sabbat», stammt von Gustave Doré, abgebildet in: Richard Evans Schultes/Albert Hofmann: Pflanzen der Götter. Die magischen Kräfte der Rausch- und Giftgewächse [Plants of the gods, übers. v. Marianne Coray und Dora Gerhard] Athenäum Verlag 1995, 91. Herman de Vries gibt eine gelungene Übersicht zu den Legenden über die Hexensalben. Hervorzuheben sind die von ihm angeführten Pflanzennamen und die Rezepte [Herman de Vries: «Über die sogenannten Hexensalben», Zeitschrift für geistbewegende Pflanzen und Kultur/Journal for mind-moving plants and cultur 1, 1991, 31-42].

2 Hierzu und zu weiteren aktuellen Fällen einer Intoxikationspsychose: Jürgen Müller: «Intoxikationspsychosen durch Atropin und Skopolamin», Nervenheilkunde (Sonderheft 3a)(16) 1997, 25; eine Darstellung der Pharmakologie und eine Übersicht über die publizierten Kasuistiken erschien 1998 in den Fortschritten der Neurologie und Psychiatrie. Im Juli 1998 verstarb in Zürich eine 20jährige Frau an einer Intoxikation, die sie sich durch einen selbstbereiteten Tee aus Bestandteilen der Engelstrompete zuzog. Fünf Frauen und drei Männer mussten mit Vergiftungserscheinungen ins Spital gebracht werden [NZZ 25./26. 7. 1998, S. 47]. Auch in Bern kam es zu einem Vergiftungsfall [Berner Zeitung 28. 7. 1998, S. 9].

3 H. J. Schneck/J. Rupreht: «Central anticholinergic syndrome (CAS) in anaesthesia and intensive care», Acta anaesthesiologica Belgica 40, 1978, 219-228. 
hinzugezogenen Notarzt wurde er in ein psychiatrisches Krankenhaus eingewiesen. Auf der Station wollte er Pilze pflücken. Er war allseits desorientiert, läppisch, dann wieder ängstlich gestimmt. Ein von ihm als Vater angegebener Mann entpuppte sich als ein weitläufiger Verwandter. Seine Haut war trocken und gerötet, die Pupillen waren weit und lichtstarr. Toxikologisch konnten Atropin und Skopolamin nachgewiesen werden. Ursächlich kam vor allem ein Getränk mit Tollkirschen in Frage.

\section{Die Familie der Solanazeen}

Die Familie der Solanazeen umfasst neben Nahrungspflanzen wie Kartoffeln, Tomaten, Paprika, Auberginen auch Tabak und eine Reihe von Rauschdrogenpflanzen. Vorwiegend als Rauschdrogen benutzt werden der Stechapfel (Datura) ${ }^{4}$, der Nachtschatten $(\text { Solanum })^{5}$, die Tollkirsche (Atropa) ${ }^{6}$, das Bilsenkraut (Hyoscyamus) ${ }^{7}$, das Tollkraut (Scopolia), der Alraun (Mandragora $)^{8}$ und verschiedene Dubiosa-Arten in Australien. Die wichtigsten Alkaloide sind Atropin und Skopolamin, deren Verhältnis stark variieren kann. Lediglich die Gattung Solanum enthält neben anderen neutralen Substanzen Saponine, Solanine und Steroidalkaloidglykoside.

4 Datura stramonium oder Weisser Stechapfel, Asthmakraut, Jimsonweed, Thornapple, Düpelsappel, Pferdegift, Dollkraut, Stachelnuss, Rauhapfel, Igelskolben wurde Fühner zufolge erst im 16. Jahrhundert durch Zigeuner aus Asien nach Europa gebracht. Snell, der bei Selbstversuchen mit Datura stramonium keine «Hexensabbat»-Phänomene verspürt hatte, bestreitet, dass der Stechapfel zur Bereitung von Hexensalben verwendet worden ist, da die Pflanze erst im 18. Jahrhundert aufgetaucht sei [H. Fühner: «Solanazeen als Berauschungsmittel. Eine historisch-ethnologische Studie», Naunyn-Schmiedebergs Archiv für experimentelle Pathologie und Pharmakologie 111,1926,281-294, und Snell,zit. nach Wolfgang de Boor: Pharmakologie und Psychopathologie, Berlin/Göttingen/Heidelberg 1956, 171].

5 Solanum dulcamara oder Bittersüsser Nachtschatten, Jelängerjelieber, Waaterwing, Saureben, Teufelsklatten, Mausholz, Zaunreben, Alpenranken, Bittersweet, Woody Nightshade und Solanum Nigrum oder Schwarzer Nachtschatten, Sautod, Black Nightshade aus der Gattung Solanum enthalten neutrale Saponine und verschiedene Steroidalkaloidglykoside [Dietrich Frohne/Hans Jürgen Pfänder: Giftpflanzen. Ein Handbuch für Apotheker, Ärzte, Toxikologen und Biologen. Mit einem Geleitwort von Otmar Wassermann, 2. durchges. Aufl., Stuttgart 1983, 215-218].

6 Atropa belladonna oder Tollkirsche, Deiwelskirsche, Dollwurz, Irrbeere, Rasewurz, Deadly Nightshade, Teufelskraut, Apfel von Sodom, tödlicher Nachtschatten [William Emboden: Narcotic plants, London 1972, 79f.].

7 Hyoscyamus niger oder Schwarzes Bilsenkraut, Bilsem, Billerkraut, Belsen, Binselkraut, Bülsen, Dollkraut, Hühnertod, Schlafkraut, Black Henbane, Henbane, Zigeunerkraut, Teufelsauge, Jusquiama noire, Schweinsbohne.

8 Mandragora officinarum oder der Alraun [Schulthes/Hofman, a. O., oben Anm. 1, 80]. 


\section{Zur Etymologie}

«Atropa» ${ }^{9}$, der botanische Gattungsname der Tollkirsche, leitet sich von der griechischen Schicksalsgöttin Atropos her: der Unabwendbaren, der Göttin des Totenreiches, die den Lebensfaden der Menschen zerschneidet. In Venetien trug ihr die mydriatische Wirkung den Namen Atropa belladonna ein: Ins Auge geträufelt, erweitert Tollkirschenextrakt die Pupille und macht auf Kosten der Sehschärfe schöne tiefe Augen ${ }^{10}$. Die deutsche Bezeichnung Tollkirsche reflektiert die Wirkung des Atropins auf das zentrale Nervensystem ${ }^{11}$.

\section{Die «Hände der Götter» in der Antike}

Ebenso wie die toxische war auch die nutzbringende Wirkung der Solanazeen schon im Altertum bekannt. In berauschenden Getränken fanden sie sich bei fast allen Völkern der Antike. Rauschtränke galten als «Hände der Götter», die den Menschen über die Sinneswelt erhoben und durch Ekstase und Traum dem Himmel näher brachten ${ }^{12}$. Bereits die klassischen Sagen berichten von tod- und heilbringenden Pflanzen. So hatten Medea ${ }^{13}$ und Kirke ihr Wissen von ihrer Mutter Hekate. Hekates Priesterinnen kannten Mandragora, Belladonna und wahrscheinlich auch Scopolia atropoides zur Erzeugung von Sinnestäuschungen und Schlaf. Auch die Wirkung des «Krautes der Kirke», womit in der griechischen Mythologie die Gefährten des Odysseus in Schweine verwandelt wurden, wird den Solanazeen, besonders dem Hyoscyamus, zugeschrieben ${ }^{14}$. Das Wissen um die zentralnervöse Wirkung

9 Papst leitet Tollkirsche über «Twalm» vom Gothischen «dwala», also «wahnsinnig», ab [G. Papst: Köhlers's Medizinalpflanzen in naturgetreuen Abbildungen mit kurz erläuterndem Texte. Atlas zur Pharmakopoea germanica, austriaca, belgica, danica, helvetica, hungarica, rossica, suedica, British pharmakopoeia, zum Codex medicamentarius, sowie zur Pharmacopoeia of the United States of America. Hrsg. v. G. Papst, Gera-Untermhaus 1887 (Reprint von Auszügen aus dem Gesamtwerk nach der dreibändigen Originalausgabe aus den Jahren 1887, 1889 und 1898), Augsburg 1997, 86-88]. Hierzu auch Frohne/Pfänder, a. O. (oben Anm. 5) 203.

10 Papst zufolge ist der Name Belladonna zuerst im 16. Jahrhundert in Venedig fassbar und hat eine Schminke aus dem roten Saft der Beeren bezeichnet. Als botanischer Begriff wurde Belladonna zuerst 1558 in den Commentarii von Matthiolus verwendet (Papst, a. O., oben Anm. 9, 86).

11 Zur Pharmakologie vergleiche Otto Gessner: Gift- und Arzneipflanzen von Mitteleuropa. Hrsg. v. Gerhard Orzechowski, Heidelberg ${ }^{3} 1974,25-36$.

12 Auch die Götter hatten ihre Orakel. «And when the anthropomorphic God dies, we have left the impersonal forces of science», bedauert Weston La Barr [zit. nach Emboden, a. O., oben Anm. 6, 78f.].

13 Hierzu die Sage von Medea und Jason: Mit Hilfe von Medeas Tränken gelingt es Jason, mit den feuerspeienden Stieren zu pflügen und das goldene Vlies zu rauben. Als Jason später Glauke heiratet, schenkt Medea das giftbestrichene Hochzeitskleid, so dass Glauke unter heftigsten Qualen stirbt.

14 Hans H. Maurer: «Toxikologie im Spiegel der Zeit», Saarl. Ärzteblatt 43, 1990, 71-80. 
der Mandragora spiegelt sich auch in einer altägyptischen Göttersage, der zufolge Hathor begann, die ganze Menschheit auszurotten ${ }^{15}$. Re setzte ihr deshalb ein Bier vor, dem der Saft der Mandragora zugefügt war. Hathor wurde vergnügt, trunken und erkannte die Menschen nicht mehr ${ }^{16}$.

In Ägypten suchte man bereits vor 3000 Jahren den geringen Alkoholgehalt des Bieres durch die Zugabe von Mandragora auszugleichen. Das medizinische Wissen der Ägypter über das Bilsenkraut ist im Papyrus Ebers festgehalten. In Babylon wusste man sich bereits im 3. vorchristlichen Jahrtausend die analgetische Wirkung der Nachtschattengewächse zunutze zu machen, indem Bilsenkrautsamen gegen Zahnschmerzen angewendet wurden.

Die älteste drogeninduzierte Geistesstörung ist von Arrhidaeus, dem Halbbruder Alexanders des Grossen, überliefert. Von Olympias, der Mutter Alexanders, mit einem Gift «schwachsinnig» gemacht, habe er dennoch nominell für sechs Jahre über Mazedonien regiert ${ }^{17}$. Nach dem Tod des wirklichen Regenten Perdikkas sei Arrhidaeus auf Befehl von Olympias im Jahr 324 v. Chr. ermordet worden. Toxische Substanzen waren in der römischen Kaiserzeit so gebräuchlich, dass Dioskurides vor beinahe zweitausend Jahren im Liber de venenis klagte:

«Die Vorbeugung gegen Gifte ist schwierig, weil die, welche heimlich Gift geben, es so anstellen, dass auch die Erfahrensten getäuscht werden. Die Bitterkeit nehmen sie den Giften dadurch, dass sie Süsses hinzufügen, und den schlechten Geruch decken sie durch Duftmittel. Sie mischen Gifte auch Arzneimitteln hinzu ... Sie tun sie in Getränke, in Wein, Suppen, in Honigwasser, in Linsengerichte und anderes, was essbar ist.» ${ }^{18}$

Dioskurides beschrieb im vierten Buch der Übersicht De materia medica dosisabhängige psychopathologische Symptome. Ihm war neben der hypnotischen auch die toxische Wirkung der Mandragora, die in höheren Dosen Erbrechen und schwerste Vergiftungserscheinungen hervorrufe, bekannt ${ }^{19}$. Hinsichtlich einer Intoxikation durch Datura stramonium beschrieb Dioskurides ebenfalls unterschiedliche Schweregrade: So riefen niedrige Dosierungen ( $1 / 2$ Drachme in Wein gelöst) angenehme Sinneseindrücke hervor. Höhere Dosierungen (2 Drachmen) führten dagegen zu Rauschzuständen

15 Louis Lewin: Die Gifte in der Weltgeschichte. Toxikologische allgemeinverständliche Untersuchungen der Historischen Quellen. [Meiner Frau, der Gefährtin eines langen Arbeitslebens, der treuen Helferin auch an diesem Werk, zugeeignet]. Reprograph. Nachdr. d. Ausg. Berlin, Springer 1920, Hildesheim ${ }^{2} 1983,33$.

16 Lewin, a. O. (oben Anm. 15) $1 \mathrm{ff}$.

17 Ebd., 1.

18 Zit. nach ebd., 1.

19 Mechthild Amberger-Lahrmann/Dietrich Schmähl (Hgg.): Gifte. Geschichte der Toxikologie. Mit 65 Abbildungen und 14 Tabellen, Berlin/Heidelberg/New York/London/Paris/Tokyo 1988, 1-46. 
bis zu drei Tagen, Dosierungen über 4 Drachmen zu einem tödlich endenden Rausch. Als Antidot kannte er Honig und Milch in grossen Mengen und anschliessendes Erbrechen ${ }^{20}$.

Galen kannte die dosisabhängige, lebensgefährliche Wirkung des Opiums, des Bilsenkrauts, der Mandragora und des Schierlings ${ }^{21}$. Mit ihm klagte Horaz:

«Diebe und Raubvögel machen mir weniger zu schaffen als jene Vetteln, die durch Zauberlieder und giftige Tränke den Menschen den Kopf verrücken.»22

Im Jahre 37 und 38 stiessen die Truppen von Antonius auf ein Kraut, das tötete, nachdem es geisteskrank gemacht hatte. Lewin führte diese mit galligem Erbrechen einhergehende Intoxikation auf ein Nachtschattengewächs, vielleicht eine Datura- oder Hyoscyamus-Art, zurück ${ }^{23}$.

Gaius Plinius Secundus d.Ä. (23-79 n. Chr.) zufolge wurde das Bilsenkraut «altercum» genannt, da es «rabies», also Tollwut, Wut, Raserei und Jähzorn, erzeuge. Plinius deutete auch bereits eine Anwendung vor chirurgischen Eingriffen $a^{24}$. Plinius und Dioskurides beschrieben einen Alraunen-Wein bzw. ein Alraunen-Brot, die nach oraler Einnahme in einen mehrstündigen ohnmachtähnlichen Schlaf fallen lassen.

Im 1. Jahrhundert nach Christus beschrieb Aretaios von Kappadozien als Nebenwirkungen der Solanazeen einen der Manie entsprechenden Zustand geistiger Störungen, der aber nicht als Manie bezeichnet werden könne, da er rascher vorübergehe.

Der Demotic Magical Papyrus von London und Leiden aus der römischen Kaiserzeit enthält folgendes Rezept, um eine Person zwei Tage schlafen zu lassen: Radix Mandragora und Liquiritiae, Hyoscyamus und Hedera je eine Unze in Wein.

\section{Mittelalterliche Narkoseverfahren}

Der spanische Bischof Isidor von Sevilla (um 570-636) warnte im 6. Jahrhundert vor dieser «herba insana», weil ihr Gebrauch wahnsinnig mache und wirre Träume hervorrufe. Auch Hildegard von Bingen (1098-1179) schrieb die toxischen und halluzinogenen Wirkungen der Solanazeen dem Teufel zu. Dennoch wusste man im 12. Jahrhundert längst, sich die anästhesierende

20 Dioskurides, Pedanius: De materia medica. Berlin 1907, zit. nach K. Dieckhöfer/Th. Vogel/ J. Meyer-Lindenberg: «Datura Stramonium als Rauschmittel», Nervenarzt 42, 1971, 431-437.

21 Lewin, a. O. (oben Anm. 15) 4 und Amberger-Lahrmann/Schmähl, a. O. (oben Anm. 19) 1-46.

22 Zit. nach Lewin, a. O. (oben Anm. 15) 4.

23 Ebd., 3f.

24 Gundolf Keil: «Spongia somnifera. Mittelalterliche Meilensteine auf dem Weg zur Voll- und Lokalnarkose», Anaesthesist 38, 1989, 543-648. 
Eigenschaft zunutze zu machen. Nach Gundolf Keil ${ }^{25}$ lassen sich bereits um 1000 n.Chr. ${ }^{26}$ eine Voll- und eine Lokalanästhesie unterscheiden. Die älteste Narkose-Anleitung aus dem 9. Jahrhundert ziele schon auf die Linderung des Operationsschmerzes: «ut sectionis dolorem non sentiant $»^{27}$. Zur Vollnarkose wurden Mohnsaft sowie ein Pressaft aus den frischen Pflanzenteilen von Alraune, Bilsenkraut (und Wasserschierling) ${ }^{28}$ verwendet; mit diesen wurde ein Schwamm getränkt und anschliessend an der Luft getrocknet ${ }^{29}$. Vor Operationsbeginn wurde der Schlafschwamm mit heissem Wasser befeuchtet und dem auf dem Rücken liegenden Patienten über Mund und Nase gelegt, so dass er durch die «spongia somnifera» atmen musste. Aus dem triefenden Schwamm in Mund und Nase tropfendes Narkosemittel wurde gastrointestinal resorbiert ${ }^{30}$. Durch Roger von Salerno ist eine Miniatur, die die Einatmung von Bilsenkrautdämpfen bei der Behandlung des Zahnwurmes darstellt, aus dem 13. Jahrhundert überliefert. Dieses Narkoseverfahren fand Eingang in das im folgenden zu besprechende Antidotarium Nicolai ${ }^{31}$. Dort suchte man bereits nach zentralerregenden Mitteln, mit denen die Narkose gesteuert werden könne. Mit frischem Fenchelsaft (Anethol bzw. d-Fenchon), in späteren Texten auch mit Essigsaft wurde ein sogenannter «Weckschwamm» getränkt, der zum Ausleiten der Narkose benutzt wurde ${ }^{32}$. Dennoch blieben Narkosezwischenfälle nicht aus. Wenngleich Guy de Chauliac noch 1363 in der Chirurgia magna die Herstellung und die An-

25 Keil, a. O. (oben Anm. 24) 645f.

26 Mechthild Amberger-Lahrmann zufolge seien die Spongia somnifera bereits in der alexandrinischen Heilkunst um 300 v.Chr. therapeutisch genutzt worden (a. O., oben Anm. 19, 12f.).

27 Gundolf Keil: «Nicolaus Salernitanus», in: Die deutsche Literatur des Mittelalters. Verfasserlexikon, begr. v. Wolfgang Stammler, fortgef. v. Karl Langosch, 2., völlig neu bearb. Aufl. unter Mitarbeit zahlreicher Fachgelehrter, hrsg. v. Kurt Ruh zusammen mit Gundolf Keil, Werner Schröder, Burghart Wachinger und Franz Josef Worstbrock, Red. Christine Stöllinger-Löser, Bd. 6 (3,4) Berlin/New York 1987, 645 (Sonderdruck).

28 Gundolf Keil: «Traktat von schlafmachenden Stücken nach der arabischen Weise (Ars somnifera)», in: Die deutsche Literatur des Mittelalters. Verfasserlexikon, Bd. $9(3,4)$ (vgl. oben Anm. 27) 1995, 997 (Sonderdruck).

29 Marguerite Baur betrachtet diese Spongia-Anwendung als Legende. Demgegenüber weist Daems auf die zur damaligen Zeit und auch heute noch bestehende unzureichende Quellenbearbeitung hin. Darüber hinaus betont Daems den Unterschied zwischen einem häufig auf irrationalen und metaphysischen Faktoren fussenden Heilmittel und einer pharmakologischen Wirksubstanz. [Willem F(rans) Daems: «Spongia somnifera. Philologische und pharmakologische Probleme», Beiträge zur Geschichte der Pharmazie 22, 1970, 25-26].

30 Diepgen und Trendelenburg wiesen von Brunn darauf hin, dass sich die Narkosewirkung nur entfalten konnte, wenn die Flüssigkeit entweder getrunken oder der Verbrennungsrauch der verbrannten Schlafschwämme eingeatmet wurde. [Walter von Brunn: «Die Stellung des Guy de Chauliac in der Chirurgie des Mittelalters», Archiv für Geschichte der Medizin, hrsg. v. d. Puschmann-Stiftung an der Univ. Leipzig unter Redaktion von Karl Sudhoff (=Sudhoffs Arch.) 12, 1920, 85-110].

31 Hierzu die kritischen Anmerkung von Brunns, a. O. (oben Anm. 30) 92-95.

32 Keil, a. O. (oben Anm. 27) 645. 
wendung der Schlafschwämme schilderte, so warnte er auf Grund tödlich verlaufener Narkosen und irreversibler Narkoseschäden doch nachdrücklich vor ihrer Anwendung. Lokalanästhesierende Verfahren führte Jesus Hali um 990 ein, als er vor operativen Eingriffen am Auge Horn- und Bindehaut durch Mohnsaft und andere Alkaloide schmerzunempfindlich machte ${ }^{33}$.

\section{Das «Antidotarium Nicolai parvum〉}

Um die Mitte des 11. Jahrhunderts brachte Konstantinus Africanus, ein Mönch aus Karthago ${ }^{34}$, Kenntnisse der arabischen Medizin und Pharmazie nach Salerno ${ }^{35}$. Aus seinen lateinischen Übersetzungen der arabischen Rezeptformelsammlungen gingen der Liber graduum, der Liber iste und das Antidotarium magnum hervor, die den Weg bahnten für das durch Nicolaus Salernitanus verbreitete Antidotarium Nicolai parvum, der bedeutendsten Formelsammlung des Mittelalters ${ }^{36}$. Von den 140 Präparaten des Antidotarium Nicolai aus der Mitte des 12. Jahrhunderts ${ }^{37}$ enthielten nicht weniger als 29 Opium, 19 Hyoscyamus und 14 Madragora; in neun waren alle drei Drogen verordnet. Über ein Drittel aller Präparate enthielten also zumindest eine der Drogen mit narkotischer oder das ZNS beeinflussender Wirkung. Bis ins 16. Jahrhundert enthielten die «populairstes» Salbenzubereitungen wie das Unguentum populeon (Pappelsalbe) neben Pappelknospen meist Papaver und Solanazeendrogen und wurden auf verschiedenste Arten appliziert: Es gab Schlafschwämme, Umschläge, Pflaster, Öle, Salben, Räucherungen, Riechmittel, Suppositorien, Tränke, Wässer, Pillen, Trochisci, Pulver, Elektuarien, Confectiones und Opiata.

\section{Die Hexensalben}

Im 17. Jahrhundert trat die therapeutische Bedeutung der Solanazeen hinter die der Opiumpräparate zurück. Als «pharmaca diabolica» wurden sie zur Grundlage der Hexensalben. Neben einer geduldeten und unterstützten weissen Magie war bereits seit dem Altertum eine schwarze Magie bekannt.

33 Ebd.

34 Werner Gaude: Die alte Apotheke. Eine tausendjährige Kulturgeschichte, Stuttgart ${ }^{2} 1986,12$ und de Vries (oben Anm. 1).

35 Zur historischen Dimension der Phytotherapie im Mittelalter vergleiche die Übersicht: Gundolf Keil: «Phytotherapie im Mittelalter», Scientiarum Historia 20, 1994, 7-38.

36 Vgl. Keil, a. O. (oben Anm. 35) 11, und Gaude, a. O. (oben Anm. 34) 12.

37 Nach Keil eine der wichtigsten, wenn nicht die wichtigste Formelsammlung mittelalterlicher Pharmazie, die bis in das 19. Jahrhundert hinein den Grundstock aller späteren Arzneibücher bildete. 
Mit ihrer Hilfe konnten Menschen geschädigt und wahnsinnig gemacht werden. Sie liess durch die Lüfte fliegen, kannte Aphrodisiaka und konnte sexuelle Gefühle hervorrufen:

«Will die Hexe zur Teufelsbuhlschaft auf den Blocksberg reiten, so zieht sie sich nackt aus, reibt sich den ganzen Körper, oder besser Anus und Genitalien, mit ihrer grünen Salbe ein, steigt in den Backtrog, nimmt Besen oder Ofengabel zwischen die Beine, und bald beginnt die nächtliche Fahrt.» ${ }^{38}$

Mit der Hexensalbe wurden die Opfer nicht nur betäubt, sondern diese konnten die imaginierte Luftfahrt, die festlichen Gelage, Tanz und Liebe so sinnfällig erleben, dass das Opfer nach dem Wiedererwachen von der Wirklichkeit des Geträumten überzeugt war. Die Hexensalben wurden am ganzen Körper, auch unter die Achseln, in den Mastdarm und wahrscheinlich auch in die Scheide ${ }^{39}$, so tief wie möglich eingebracht ${ }^{40}$. Fühner zufolge stellten die Hexensalben ein Berauschungs- und Genussmittel des armen Volkes dar, dem kostspieligere Genüsse versagt waren ${ }^{41}$. Die Wirkungen der schwarzen Magie wurden dem Satan zugeschrieben ${ }^{42}$. Der Aberglaube lehrte, dass beispielsweise die Mandragora unter besonderen Vorsichtsmassnahmen geerntet werden müsse ${ }^{43}$, wie bereits von Theophrast im 3. vorchristlichen Jahrhundert beschrieben: Es wurden Kreise um die Pflanze gezogen, dann nach Westen gewandt, das oberste Wurzelstück abgeschnitten. Der Rest der Wurzel wurde erst nach bestimmten Tanzschritten und nach dem Aufsagen einer bestimmten Formel herausgezogen. Die Pflanze war so gefürchtet, dass auch ein Hund an ihr festgebunden wurde, damit er sie ausriss und später verendete, wie die Volkssage lehrte. Nach mittelalterlicher Auffassung musste man sich vor dem Ausgraben des Alrauns die Ohren verstopfen, um von dem fürchterlichen Schrei, den der Alraun ausstosse, nicht getötet zu werden. Der Fabel zufolge wachse der Alraun unter dem Galgen aus dem Harn oder Sperma eines gehängten Diebes. Dies trug dem Alraun im Volksmund die

38 Fühner, a. O. (oben Anm. 4) 286.

39 Schultes/Hofmann, a. O. (oben Anm. 1) 90.

40 Zit. nach Lewin, a. O. (oben Anm. 15) 12.

41 Fühner, a. O. (oben Anm. 4) 286.

42 Lewin, a. O. (oben Anm. 15) 10.

43 William Emboden [a.O., oben Anm. 6, 132f.] zufolge findet sich eine Variante der Legende in den meisten grossen Herbarien der Renaissance: «Mandrakes are to be found under gallows, for they issue from the ground/ where the semen of a hanged man has fallen. If a mandrake be pulled/ from a ground, it will come forth with such a shriek of horror that the/ collector upon hearing the cry will fall dead. However, if the herb be tied/ at the base of its rosette with a rope, and the other end of the rope be/ tied to the neck of a dog, the dog may be persuaded to run from the/ plant by his master's call. Upon lurching forward, the dog will pull the/ plant from the ground and as a consequence will fall dead from the terrible/ scream. Collectors, however, will have taken the precaution of stopping/ up their ears with wool, and to have cut around the plant with a double/ edged sword while murmuring incantations to protect them from harm.» Hierzu Schultes/Hofmann, a. O. (oben Anm. 1) 89. 
Bezeichnung «Galgenmännlein» ein ${ }^{44}$. Auch Shakespeare wusste sich die Legende in Romeo and Julia, König Heinrich VI. und in Macbeth zunutze zu machen ${ }^{45}$.

In der Grauzone zwischen schwarzer und weisser Magie beschrieb Giambattista della Porta in seiner Magia naturalis, wie man einen Menschen beispielsweise zur Belustigung bei Gelagen für einen Tag lang verrückt machen könne, wozu Mandragora, Solanum maniacum Datura, Belladonna und Hyoscyamus verwendet wurden ${ }^{46}$. Ihm zufolge konnte den Vergifteten leicht suggeriert werden, dass sie in Tiere verwandelt worden seien, so dass sie Schwimmbewegungen machten, mit Flügeln schlugen wie Gänse und mit Hörnern stiessen wie Ochsen ${ }^{47}$. Die Kenntnisse über Pflanzengifte machte man sich zu den verschiedensten Zwecken dienstbar. So wussten Pferdehändler, dass selbst die elendste Schindmähre feurig wie ein Vollblüter werde, wenn man ihr ein paar zusammengerollte Daturablätter in den Mastdarm steckte ${ }^{48}$.

\section{Pocula amatoria}

Bei der Zubereitung von «Hexensalben» und «Hexentränken» spielte das Bilsenkraut ${ }^{49}$ eine herausragende Rolle. In den mittelalterlichen Badestuben wurde Bilsensamen auf den Ofen gestreut, woraufhin «Männlein und Weiblein aneinanderstiessen». Die «pocula amatoria» wurden bereits von Justinian in der Lex Cornelia den magischen Künsten gleichgestellt und seit dem 13. Jahrhundert durch Kaiser Friedrich II. streng geahndet. Ein Gutachten durch die Leipziger Fakultät bestätigte noch 1697 ihre Wirksamkeit. Noch im 18. Jahrhundert sah das allgemeine Preussische Landrecht strenge Strafen für die Liebestränke vor ${ }^{50}$.

Bereits in den antiken Kulturen wurde mit den Solanazeen Bier verstärkt. Bediente man sich in Ägypten des Alrauns, in Russland und in China des Stechapfels, so war später in Mitteleuropa vorwiegend das Bilsenkraut ge-

44 Amberger-Lahrmann/Schmähl, a.O. (oben Anm. 19) 8-13.

45 In Romeo und Julia (IV. Akt, 3. Szene) «shrieks like mandrakes torn out of the earth»; König Heinrich VI. (2. Teil, III. Akt, 2. Szene) «Would curses kill, as doth the mandrake's groan»; Macbeth «... the insane root that takes the reason prisoner». Emboden, a. O. (oben Anm. 6) 133.

46 Johann Baptist Porta (1535-1615), Magiae naturalis libri 20, Neapel 1589, 151, zit. nach Fühner, a. O. (oben Anm. 4) $283 \mathrm{ff}$.

47 Fühner, a. O. (oben Anm. 4) $283 \mathrm{f}$.

48 Frohne/Pfänder, a. O. (oben Anm. 5) 206.

49 Hyoscyamus niger, das Schwarze Bilsen- oder Schlafkraut [ebd., 208].

50 Fühner, a. O. (oben Anm. 4) und Lewin, a. O. (oben Anm. 15) 16-22. 
bräuchlich, was noch die Ortsnamen Bilsengarten, Bilsensee, Bilsdorf, Bilsen in den Niederlanden und Pilsen in Böhmen verkünden ${ }^{51}$.

Die Verwendung des Bilsenkrauts zur Bierverstärkung war so ausgiebig, dass gesetzlich entgegengetreten werden musste. So sah die Eichstätter Polizeiordnung aus dem Jahre 1507 eine Strafe von 5 Gulden vor und gebot, dass der Brauer keine Bilsensamen und andere «den Kopf tollmachenden Stücke und Kräuter» ins Bier mischen sollte. Nach der bayerischen Landund Polizeiordnung von 1649 war es zwar unverboten,

«ein wenig Salz, Krammetbeer und Kümmel ins Bier zu nehmen; wer aber andere Kräuter und Samen, fürnehmlich Bilsen, in das Bier tut, der soll, wie auch der Verkäufer solcher Kräuter, nach Ungnaden gestraft werden.» ${ }^{52}$

Johann Theodor Tabernaemontanus droht in seinem Kräuterbuch aus dem Jahr 1664:

«Die aber mit Bilsensamen und anderen dergleichen schädlichen Dingen das Bier stärken, sollen verworfen und verdammt werden, [...] diejenigen, so mit dergleichen schädlichen Künsten das Bier verfälschen, als abgesagte Feind des menschlichen Geschlechts, als Dieb und Mörder an Leib und Leben» gestraft werden. «Biere mit Bilsensamen soll Niemand trinken, denn diejenigen, so das Leben verwirkt haben, denn sie bringen Hirnwüten, Unsinnigkeit und bisweilen den gähen Tod.» ${ }^{53}$

Die Bezeichnung «Altsitzerkraut» spiegelt wider, dass man sich mit Bilsenkraut auch untätig auf einer Bank sitzender Greise entledigte. In Teilen Litauens führt die Pflanze Hyoscyamus scopolia noch heute den Namen Altsitzerkraut.

\section{Mordgifte}

Die bei hoher Dosis tödliche Wirkung der Solanazeen machte die Pflanzen in gleicher Weise für Mordzwecke wie für Exekutionen im Strafvollzug tauglich. Luzerner Stadtrechnungen aus dem 16. Jahrhundert belegen den Bezug von Bilsenkrautsamen durch Henker. Zahlreiche unerklärliche Todesfälle in den obersten Kreisen der französischen Gesellschaft 1670 entpuppten sich als grossangelegte Giftmordtragödien, die den Solanazeenalkaloiden angelastet wurden. Ludwig XIV. liess für die Untersuchung eine eigene 'Chambre ardente' oder den 'Cours des poisons' einrichten. Zwischen 1679 und 1682 wurden über 400 Personen in die Verfahren einbezogen, davon 87 als Zauberinnen und Giftmischer zum Tode verurteilt. Als «Hoflieferantin» für Gifte und Liebestränke, zu denen sie Bilsenkraut, Stech-

51 Fühner, a. O. (oben Anm. 4) 292f.

52 Ebd., 293.

53 Johann Theodor Taberaemontanus: Kräuterbuch, Basel 1664, 637, zit. nach Fühner, a. O. (oben Anm. 4) 293f. 
apfel und Spanische Fliegen, bei Gifttränken auch das Arsenik verwendete,

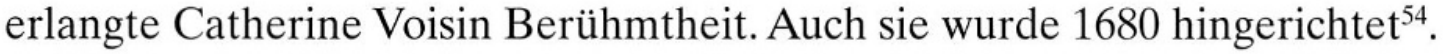
Shakespeare ${ }^{55}$ schätzte Skopolamin- und Bilsenkrautzubereitungen als «Mordgift». Er liess den Vater Hamlets durch 'Hebone' umbringen, eine Substanz, die heute als Bilsenkraut gedeutet wird. Dabei war ihm bereits die gute Resorption des Alkaloids im Ohrenbereich bekannt ${ }^{56}$. Doch auch in der historischen Vorlage zum Macbeth sollen Solanazeengifte verwendet worden sein ${ }^{57}$ : Als Duncan I. vom Dänenkönig Sven bei Perth belagert wurde, liess er den hungernden Belagerern viel Wein, Brot und ein mit dem Saft des schlafmachenden Nachtschattens, solanum somniferum, versetztes Getränk bringen. Während die Dänen dem Trank zusprachen, liess Duncan Macbeth mit seinen Truppen heimlich in die Stadt. Nur wenige der überraschten Dänen liefen, vom Kriegsgeschrei der Schotten aufgeweckt, verwirrt umher und wurden getötet. Die meisten jedoch starben unmittelbar an der Vergiftung. Sven entkam mit einigen wenigen Überlebenden mit dem Schiff.

Neben der dämpfenden und schliesslich todbringenden fand auch die anregende und enthemmende Wirkung Anwendung. So wurden die Solanazeen im 17. Jahrhundert auch als Wahrheitsdrogen verwendet, wozu insbesondere die atropinhaltigen Essenzen zugeschriebene Steigerung der Suggestibilität beitrug $^{58}$.

\section{Die Entdeckung der Wirksubstanzen und ihre klinische Anwendung}

In der zweiten Hälfte des 19. Jahrhunderts erkannten Ladenburg und Schmidt die bis dahin als Atropin, Belladonnin, Daturin, Hyoscyamin, Duboisin, Scopolein bezeichneten Substanzen als Gemische vorwiegend aus Atropin und Hyoscyamin. Später konnte Ladenburg ${ }^{59}$ noch Hyoscin isolieren, das im folgenden grosse Bedeutung als Heilmittel erlangte ${ }^{60}$. Edlefsen hob 1881 die schmerzstillende Wirkung hervor. Rudolf Gnauck überprüfte diese Ergebnisse ohne Erfolg, stellte jedoch eine beruhigende und schlafanstossende Wirkung fest. Er konnte zuerst bei Gesunden, dann bei Geistes-

54 Maurer, a. O. (oben Anm. 14) 71-80 und Lewin, a. O. (oben Anm. 15) 46-53.

55 Bereits zuvor wurde darauf hingewiesen, dass Shakespeare auch der sich um die Mandragora rankenden Legenden bediente, und zwar in Romeo und Julia, König Heinrich VI. und Macbeth. Hierzu auch Emboden, a. O. (oben Anm. 6) 133.

56 Eelco Huzinga: «Murder Through the ear», Practica oto-rhino-laryngica 33, 1971, 361-365.

57 Zit. nach Lewin, a. O. (oben Anm. 15) 53.

58 Erst im 20. Jahrhundert löste das Amytal die Nachtschattenalkaloide zur «Geständnisgewinnung» ab. De Boor, a. O. (oben Anm. 4) 28.

59 Albert Ladenburg: «Die natürlich vorkomenden mydriatisch wirkenden Alkaloide», J. Liebig's Annalen der Chemie 206, 1881, 274-307.

60 August Sohrt: Pharmacotherapeutische Studien über das Hyoscin, Med. Diss. (Dorpat) 1886. 
kranken eine «schlafmachende» und beruhigende Wirkung bei innerlicher und subcutaner Anwendung belegen ${ }^{61}$. August Sohrt untersuchte 1886 in seiner Dorpater Dissertation die Wirkung des Hyoscins auf das vegetative Nervensystem und setzte die Substanz mit Erfolg bei aufgeregten und tobsüchtigen Geisteskranken ein. Ihm zufolge wirke Hyoscin auf Gesunde narkotisierend, beeinflusse aber im Gegensatz zu Atropin die elektrische Erregbarkeit des Gehirns nicht ${ }^{62}$. Emil Kraepelin, Lehrer Sohrts, bezeichnete das von Gnauck erstmalig bei Geisteskranken angewendete Hyoscin als ein für die irrenärztliche Behandlung recht wertvolles Mittel ${ }^{63}$. Dieses Alkaloid erzeuge in subcutaner Gabe von 0,0005-0,001g mit unübertroffener Sicherheit einen nach 10-15 Minuten eintretenden tiefen Schlaf. Eine Vergiftung werde eingeleitet durch Eingenommenheit des Kopfes, Trockenheit im Halse, Schwere der Zunge, Unsicherheit beim Gehen oder eine mehrere Tage, selbst Wochen lang andauernde, hochgradige Pupillenerweiterung. Grössere Gaben könnten zu Übelkeit, Unregelmässigkeit des Pulses, Atmungsbehinderung, Gesichtstäuschungen, selbst Delirien und Kollapszustände führen, doch hätten hier vielleicht gelegentlich Verunreinigungen eine gewisse Rolle gespielt. Kraepelin selbst habe jedenfalls niemals bedrohlichere Erscheinungen beobachten können, obwohl er das Mittel durch eine Reihe von Jahren überaus häufig habe anwenden müssen. Nach dem Erwachen bestehe gewöhnlich das Gefühl von Abgeschlagenheit und ein leichter Druck im Kopfe, der sich meist bald verliere. Hyoscin sei demnach überall dort, wo dringend und rasch Beruhigung und Schlaf verschafft werden müsse, zuverlässig und meist ohne erhebliche Nachteile wirksam. Bei längerem Gebrauch trete eine gewisse Gewöhnung ein. Entziehungserscheinungen träten jedoch nicht auf ${ }^{64}$.

\section{Der «Sommer der Blumenkinder»}

In der zweiten Hälfte des 20. Jahrhunderts erfuhren die Solanazeenalkaloide während der Studentenbewegung eine Renaissance. Im sogenannten «Sommer der Blumenkinder» beobachtete Teitelbaum 1967 im Einzugsgebiet der Universität Colorado innerhalb weniger Monate 20 Vergiftungsfälle mit

61 Rudolf Gnauck: «Ueber die Anwendung des Hyoscin bei Geisteskranken», Charité-Annalen $7,1881,448-465$.

62 Sohrt, a. O. (oben Anm. 60).

63 Emil Kraepelin: Psychiatrie. Ein Lehrbuch für Studierende und Ärzte, Bd. I. Allgemeine Psychiatrie, Leipzig ${ }^{7} 1903,398$.

64 Kraepelin, a. O. (oben Anm. 63) 398; hierzu auch Eugen Bleuler: Lehrbuch der Psychiatrie, 10. Aufl. umgearbeitet von Manfred Bleuler (Zürich), mit 121 Abbildungen, Berlin/Göttingen/Heidelberg 1960,152-166. 
Datura stramonium. Auf der Suche nach verfügbaren, preiswerten und legalen Drogen waren amerikanische Studenten auf das bewährte Asthmamittel «Asthmador», ein aus den Blättern von Datura stramonium gewonnenes Anticholinergikum, das in Form von Pfeifentabak, Zigaretten und Pulver gehandelt wurde und als inhalatives Asthmamittel gebräuchlich war, gestossen $^{65}$. Während Dean 1963 drei Intoxikationsfälle publizierte, berichtete Goldsmith 1968 von zwei Vergiftungsfällen, von denen einer sechs Tage stationär behandelt werden musste ${ }^{66}$. Nach Deutschland gelangten die Datura stramonium-Intoxikationen wenig später, ausgelöst durch Brosig's Asthmazigaretten. Im Oktober 1969 berichteten Engelmeier und Finke von zwei Hippies, die während eines «Pop und Blues»-Festivals je vier Asthmazigaretten konsumiert hatten und nacheinander zur Aufnahme kamen. Beide Patienten waren nicht kontaktfähig, verkannten die Situation und halluzinierten. Ihre Sprache war zum unverständlichen Wortsalat zerfallen ${ }^{67}$.

\section{Die Enthüllung der Mordgifte}

Die zunehmende Verbreitung der Solanazeenalkaloide verliehen ihnen im 19. und 20. Jahrhundert auch zunehmend forensisches Gewicht ${ }^{68}$. Angesichts der freien Verfügbarkeit und bei nur geringem Aufklärungsrisiko entzog sich eine erhebliche Dunkelziffer von Morden und Mordversuchen der Aufklärung, bis in den Prozessen um Dr. Buchanan und Dr. Crippen die Toxikologie der Solanazeen erstmals zur Urteilsfindung führte.

Am 7.6.1892 wurde der knapp 30jährige New Yorker Arzt Dr. Robert W. Buchanan verhaftet: Er hatte seine Frau Annie Sutherland, eine über 50jährige, beleibte, ordinäre Bordellbesitzerin, am 23. April 1892 mit Morphium vergiftet. Da er die bei Morphinen auftretende Miosis durch Atropin kaschierte, wurde lange eine natürliche Todesursache vermutet ${ }^{69}$.

Erstmals wurde in dem Aufsehen erregenden Mordprozess gegen Dr. Hawley Harvey Crippen 1910 eine tödliche Konzentration Hyoscin in jedem

65 Zit. nach K. Dieckhöfer/Th. Vogel/J. Meyer-Lindenberg: «Datura Stramonium als Rauschmittel», Nervenarzt 42, 1971, 431-437.

66 Goldsmith beschrieb die Symptomatik überaus anschaulich: «hot as a hare, blind as a bat, dry as a bone, red as a beet and mad as a wet hen.» Stephen R. Goldsmith/Ira Frank/J.Thomas Ungerleider: «Poisoning from ingestion of a Stramonium-Belladonna Mixture. Flower power gone sour», JAMA 204, 1968, 169-170.

67 Max-Paul Engelmeier/Jobst Finke: «Stechapfelrausch», Pharmakopsychiatrie. Neuropharmakologie 3, 1970, 248-253.

68 Jürgen Thorwald: Das Jahrhundert der Detektive. Weg und Abenteuer der Kriminalistik, Band 3: Handbuch für Giftmörder, München/Zürich 1968, 41-45.

69 A. C. Moffat: «Forensic Pharmacognosy - Poisoning with plants», Journal of the Forensic Science Society 20, 1980, 103-109, und Thorwald, a. O. (oben Anm. 68) 80-94. 
einzelnen Organ seiner von ihm vergifteten Frau nachgewiesen. Bernhard Spillsbury legte mit seinen histologischen Analysen die Grundlage zur forensischen Pathologie. Dr. William Henry Willcox wies an Hand des isolierten Hyoscins in den Leichenresten nach, dass Crippen die gesamten $300 \mathrm{mg}$ Hyoscin, die er sich zwei Wochen vor dem Tod seiner Frau besorgt hatte, verabreicht hatte ${ }^{70}$. Dabei führte er Kristallisation und Schmelzpunktbestimmung in die forensische Pharmakologie ein. Erst seither birgt die Vergiftung durch Skopolamin ein gewisses Entdeckungsrisiko.

Als sich Verdächtigte damit verteidigten, sie hätten Tollkirsche nur verabreicht, um einen Aufgebrachten zu beruhigen, erlangte die quantitative Bestimmung der verabreichten Giftmenge zunehmende toxikologische Bedeutung. 1931 behauptete eine eines Gattenmordes Verdächtigte, sie habe elf Tollkirschen zu einem Heidelbeergericht gegeben, um den Gatten, der in einem Wutanfall die Familie bedrohte, zu beruhigen. Nachdem die im Magen-Darm-Trakt verbliebenen Tollkirschensamen gezählt und die Giftmenge bestimmt war, konnte die verabreichte Menge auf 25-30 Beeren beziffert werden ${ }^{71}$. Nicht nur die letale, sondern auch die sexuell stimulierende und wehrlos machende Wirkung macht man sich in krimineller Intention zunutze. Betz berichtete 1991 von einem 34jährigen Gärtner, der eine weibliche Bekannte und zwei mit dieser befreundete Studenten mittels Stechapfelsamenkörner wehrlos machte. Anschliessend habe er sie unvollständig entkleidet und sexuelle Handlungen an ihnen vorgenommen. Erwacht aus mehrstündiger Bewusstlosigkeit, waren die Männer nur teilweise bekleidet, wobei der eine die Hose des anderen trug ${ }^{72}$. Betz schrieb insbesondere dem Zustand weitgehender Wehrlosigkeit, welcher trotz erhaltener Kritikfähigkeit eine effiziente Gegenwehr unmöglich mache, forensische Bedeutung $\mathrm{zu}^{73}$. Dennoch werden Solanazeenvergiftungen auch heute noch häufig nicht geahndet, da die rauschähnliche Symptomatik auf eine zum Teil gleichzeitig bestehende Alkoholintoxikation oder auf eine andere Vergiftung zurückgeführt und infolgedessen die Substanz nicht nachgewiesen wird ${ }^{74}$. Reine Atropinvergiftungen in krimineller Absicht sind Berg zufolge selten, da

70 J. R. Maltby: «Criminal Poisoning with anaesthetic Drugs: Murder, Manslaughter, or not guilty», Forensic Science 6, 1975, 91-108.

71 Steffen Berg/K. Fischer: «Quantitative Aussagen bei einer Gruppenvergiftung (Mordversuch) mit Atropin», Deutsche Zeitschrift für Gerichtliche Medizin 53, 1962, 44-50.

72 Peter Betz/Jörk Janzen/Gabriele Roider/Randolph Penning: «Psychopathologische Befunde nach oraler Aufnahme von Inhaltsstoffen heimischer Nachtschattengewächse», Archiv für Kriminologie 188, 1991,175-182.

73 Betz et al., a. O. (oben Anm. 72) 175-182.

74 W[alter] Krauland/E. Klug, «Kriminalistische Aspekte bei der Aufklärung von Alkaloidvergiftungen», Münchner medizinische Wochenschrift 120, 1978, 1493-1496. 
die erforderliche Substanzmenge nur Ärzten und Apothekern zugänglich sei $^{75}$. Er beschrieb eine Atropinvergiftung von sechs Personen im Haushalt eines Apothekers: Eine 26jährige, drogenabhängige Kandidatin der Pharmazie gestand später, dass sie von ihrem Arbeitgeber beim Diebstahl von Preludin erwischt worden war und sie mit Hilfe der Drogen die Fahrt des Apothekers zur Apothekerkammer verhindern wollte ${ }^{76}$.

Noch immer wird Skopolamin zu Verbrechen benutzt: Eine 43jährige Frau war nach einem auf einer Autobahnraststätte eingenommenen Tee verwirrt, umtriebig und zeigte eine resonanzlose bis gereizte Stimmung. Die Patientin nestelte an ihrer Kleidung. In der toxikologischen Untersuchung konnte nur Skopolamin nachgewiesen werden, so dass eine pflanzliche Intoxikation nicht in Frage kam.

Ein anderer Patient war nach einem auf derselben Raststätte eingenommenen halben Liter Milch verwirrt und lief nur mit seiner Unterwäsche bekleidet, Blumen pflückend auf dem Rastplatz umher, wobei er versuchte, aus der Zapfsäule Benzin zu trinken. Währenddessen wurde sein LKW aufgebrochen und ausgeraubt. Toxikologisch konnten erst bei gezielter Nachuntersuchung geringe Skopolaminmengen nachgewiesen werden ${ }^{77}$. Wenngleich die verwendeten Gifte erkannt wurden, blieben auch diese Taten ungeahndet.

Intoxikationspsychosen durch Nachtschattenalkaloide sind noch immer eine wichtige Differentialdiagnose bei akut psychotischen Patienten. Wenn sie sicher auch nicht zu den häufigen Krankheitsbildern zählen, so zwingen die auch in jüngster Zeit aufgetretenen Vergiftungsfälle in Zürich und Bern ebenso wie die vier innerhalb eines Jahres in Homburg diagnostizierten Fälle zur Auseinandersetzung mit diesen jahrhundertealten Rauschdrogen ${ }^{78}$.

75 Werner Grab: «Kriminelle Atropinvergiftung», Deutsche Zeitschrift für Gerichtliche Medizin 40, 1951, 641-648, zufolge wurde in der ersten Hälfte des 20. Jahrhunderts nur ein einziger Fall beschrieben. Auch die Zusammenstellung von Falck aus der Literatur von 1867-1879 erwähnt nur eine Wärterin, die einem Hospitalbeamten in Milch gelöstes Atropin beibrachte. Grab berichtete 1951 über eine ärztliche Hilfsperson, die ihre Vermieterin um eine wertvolle Brillantnadel berauben wollte und Atropin in den Tee mischte. Erst durch die Untersuchung am isolierten Froschherz, wobei Atropin die Wirkung des inotropen Acetylcholins antagonisiert, konnte die Substanz nachgewiesen werden.

76 Berg/Fischer, a. O. (oben Anm. 71).

77 Bärbel Ziegler/Werner Tönjes: «Scopolamin-Intoxikation als Ursache akuter paranoidhalluzinatorischer Psychosen», Psychiatrische Praxis 18, 1991, 21-24.

78 Im Sommer 1998 kam es in Zürich und Bern erneut zu schweren Vergiftungsfällen, die z.T. intensiv medizinisch behandelt werden mussten (vgl. oben Anm. 2). 